\section{Muskelrelaxantien: Anaphylaxie-Risiko unter der Narkose}

$\mathrm{B}$ ei einer von 6000 Narkosen tritt ein Zwischenfall auf. Abgesehen von möglichen pharmakologischen Arzneimittelnebenwirkungen stehen dabei anaphylaktoide Reaktionen im Vordergrund. Unklar ist bislang, welche Rolle eine atopische Diathese spielt; gerätselt wird auch, warum in bis $\mathrm{zu} 90 \%$ Frauen betroffen sind.

Beispielhaft ist der Fall einer 52jährigen Patientin, die innerhalb von sechs Jahren zwei Narkosezwischenfälle hatte: 1990 kam es während eines gynäkologischen Eingriffs zu einer bronchialen Obstruktion sowie zu Tachykardie und Kammerflimmern. 1996 zog die Entfernung eines Lipom-Rezidivs außer einer Urtikaria einen Herz-Kreislauf-Stillstand nach sich; die Patientin konnte aber erfolgreich reanimiert werden.

Eine ausführliche Prick-Testung in der Dermatologischen Klinik und Poliklink der Universität München ergab eine deutlich positive Reaktion auf die Muskelrelaxantien Pancuronium, Atracurium und Vecuronium, weiterhin zeigte sich eine Senisibilisierung gegenüber Naturlatex.

Allergische Reaktionen auf Muskelrelaxantien sind laut Silke Michelsen, LMU München, für 70\% aller Narkosezwischenfälle verantwortlich. Hauptauslöser sind Suxamethonium und Vecuronium, seltener Pancuronium. Viele dieser Muskelrelaxantien, z.B. Suxamethonium und Pancuronium, gehören zur Stoffklasse der quartären Ammonium-Salze-Verbindungen, die oft auch Bestandteile von Tensiden sind. An diesem Punkt setzt eine mögliche Hypothese an, die einen Anhaltspunkt für die auffällige Häufung von Narkose-Zwischenfällen beim weiblichen Geschlecht liefert: Vermehrt im Haushalt tätige Frauen sensibilisieren sich möglicherweise durch den Kontakt mit Tensiden und Medikamenten, die quartäre Ammonium-Salze enthalten.

Vermehrt treten allergische Reaktionen auch unter Injektionsanästhetika wie z.B. Thiopental auf. Narko-Analgetika und Substanzen der Prämedikation hingegen führen eher selten zu Zwischenfällen.

Bei der ambulanten Vorstellung eines betroffenen Patienten sollte daher stets eine gründliche Anamnese erhoben sowie das Narkoseprotokoll angefordert werden. Blutproben, die während des Zwischenfalls abgenommen wurden, können hilfreiche Details hinsichtlich der Mastzell-Degranulation liefern. Vier bis sechs Wochen nach dem Zwischenfall sollten Hauttests vorgenommen werden, die möglichst alle für die Narkose eingesetzten
$\mathrm{D}$ as Thema Naturlatex-Allergie erhitzt immer wieder die Gemüter. Die Zahlenangaben für Angehörige von Gesundheitsberufen klaffen häufig weit auseinander. Ein Grund dafür: Die klinische Diagnostik wird nur unzureichend bemüht; häufig fehlen Pricktestungen und IgE-Messungen. Dieses Manko zu beseitigen, hat sich eine Arbeitsgruppe der Dermatologischen Klinik und Poliklinik der Universität München zur Aufgabe gestellt. Unter der Leitung von B. Przybilla, München, führen Dermatologen am Klinikum Innenstadt eine vom bayerischen Staatsministerium für Landesentwicklung und Umweltfragen geförderte Studie durch, um die Häufigkeit von Allergien gegen Naturlatex zu ermitteln.

Untersucht werden alle Berufsgruppen des Klinikums: von Ärzten über Labor- und Pflegepersonal bis hin zu Verwaltungsangestellten. An erster Stelle steht eine Fragebogen-gestützte, ausführliche Anamnese; gegebenenfalls werden Prick-Testungen vorgenommen sowie die IgE-Titer bestimmt. Bei auffälligen Testbefunden steht den Probanden die Möglichkeit einer epikutanen Provokation und einer Lungenfunktionsprüfung zur Verfügung.
Medikamente einschließen. In geringen Dosen und unter Beobachtung sind auch Provokationstests möglich.

Für den Wert eines Allergiepasses kommt es besonders darauf an, die risikoreichen Substanzen zu definieren und Ausweichpräparate zu nennen. Gegebenenfalls läßt sich auch eine Pharmakoprophylaxe empfehlen: z.B. Prednison zur peroralen Gabe 13, 7 und 1 Stunde vor einem geplanten Eingriff plus ein Antihistaminikum, das 1 Stunde präoperativ verabreicht wird. Alternativ eignen sich auch $\mathrm{H}_{1}$ und $\mathrm{H}_{2}$-Rezeptorblocker 10 bis $15 \mathrm{Mi}$ nuten präoperativ.

Quelle: Vortrag von Silke Michelsen, LMU München, im . lergologie“, Klinikum Großhadern, München, 28.1.1998.

\title{
Klinikstudie zur Naturlatex-Allergie: 18\% der Ärzte betroffen
}

Bislang liegen die Daten von 370 Klinikmitarbeitern vor. Die Gesamtprävalenz betrug $11 \%$. Ärzte waren mit $18 \%$ am häufigsten, Verwaltungsangehörige erwartungsgemäß am seltensten betroffen. Bei 14 Personen war eine Sensibilisierung gegen Naturlatex nachweisbar, ohne daß ein klinisches Korrelat vorlag. Von 27 Personen mit einer manifesten Allergie hatte die Mehrzahl bereits seit mehreren Jahren über Beschwerden geklagt - ein Drittel innerhalb der ersten fünf Berufsjahre. Das Symptomspektrum reichte von Kontakt-Urtikaria über Rhinokonjunktivitis bis hin zu Asthma bronchiale.

Der wesentliche Grund der Zunahme von Naturlatex-Allergien dürfte die vermehrte Billigproduktion von Handschuhen sein: Gespart wird am kostenintensiven Spülen der fertigen Handschuhe; dadurch gelangen beim späteren Gebrauch mehr Allergene in die Raumluft. Im Sinne der Primärprävention sollten daher Handschuhe ohne das allergenverschleppende $\mathrm{Pu}-$ der sowie mit möglichst geringem Latexgehalt verwendet werden.

(ro) Quelle: Vortrag von Pia Schöpf, LMU München, im Rahmen
der 18. Fortbildungsveranstaltung „,Angewandte Allergologie“, Klinikum Großhadern, München, 28.1.1998. 